\title{
Non-steady Supersonic Flow About Pointed Bodies of Revolution
}

by W.H. Dorrance

\author{
Project MX-794 \\ USAF Contract W 33-038-ac-14222
}

Willow Run Research Center

Engineering Research Institute

University of Michigan

UMM-80. November 1950 
on $8 n$ UMRO968 
List of Figures

Symbols and Nomenclature

Greek Symbols

I Summary

II Introduction

A. The Non-steady Source-sink Potential

B. The Non-steady "Doublet" Potential

C. Boundäry Conditions

IV Normal Force and Pitching Moment Coefficients

V The Low Frequency Oscillations and Steady Motion

A. Harmonic Pitching About the Point

B. Harmonic Normal Oscillations in Pitch

C. Steady Pitching About the, Point

D. Steady Angle of Attack

VI Example Results for Two Bodies of Revolution

Appendix A

Appendix B

Appendix C

References

30

Distribution 
UMM-80

\section{LIST OF FIGURES}

No.

$1 \quad$ Cylindrical Coordinates and Orientation of Body of Revolution

2a Harmonic Pitching About $\mathrm{X}_{\mathrm{O}}$

$2 b$ Harmonic Normal Oscillations in Pitch

2c Steady Pitching About $\mathrm{X}_{\mathrm{O}}$

2d Steady Angle of Attack

3 Values of $\mathrm{C}_{\mathrm{N}_{\dot{\alpha}}}$ and $\mathrm{C}_{\mathrm{M}}$. for Body (a) 20

4 Values of $C_{N}$ and $C_{M}$ for Body (a) 20

5 Values of $\mathrm{C}_{\mathrm{N}}, \mathrm{C}_{\mathrm{M}_{\alpha}}$ for Body (a) 22

6 Values of $\mathrm{C}_{\mathrm{N}_{\mathbf{q}}}$, $\mathrm{C}_{\mathrm{Mq}}$ for Body (a) 22 
c

$\mathrm{C}_{\mathrm{n}}$

$\mathrm{Cm}_{\mathrm{m}} \mathrm{SL}$

$c_{n i,} c_{m i}=\frac{\partial c_{n}}{\partial\left(\frac{\partial L}{U_{0}}\right)}, \frac{\gamma c_{m}}{\partial\left(\frac{\partial L}{U_{0}}\right)}$

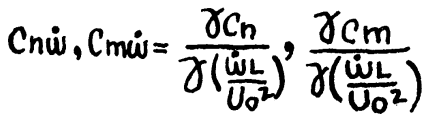

$c_{n q}, c_{m q}=\frac{\gamma c_{n}}{\gamma\left(\frac{q L}{U_{0}}\right)}, \frac{\gamma c m_{m}}{\gamma\left(\frac{q_{L}}{u_{0}}\right)}$

$c_{n}, c_{m}=\frac{\gamma c_{n}}{\partial \alpha}, \frac{\gamma c_{m}}{\partial \alpha}$.

$c p=-2\left(\frac{\varphi_{2 x}}{U_{0}}+\frac{\varphi_{2 t}}{U_{0}^{2}}\right)$
= local velocity of sound

= normal force coefficient

$=$ pitching moment about $\mathrm{x}_{\mathrm{O}}$ (positive in stall)

dimensionless non-steady pitching stabil-

ity derivatives

dimensionless normal acceleration stabil-

ity derivatives

dimensionless steady pitching stability derivatives

incremental lifting steady aerodynamic coefficients

pressure coefficient

$\overline{f(s)}=\int_{0}^{\infty} \exp \{-s x\} f(x) d x=$ Laplace transform of $f(x)$

$\mathrm{K}_{\mathrm{O}}(\mathrm{z})$

= modified Bessel Function of second kind with modulus $z$

$k=\frac{M}{\beta} \frac{\omega r}{U_{0}}$

= reduced frequency

I

M

$\mathrm{p}$

qo

q

$R(x)$

$S$

$S(x)$

t

$\mathrm{U}_{\mathrm{O}}$

$\overrightarrow{\mathrm{V}}$

$\mathrm{w}(\mathrm{x}, \mathrm{t})$
= length of body of revolution

= free stream Mach number

= local static pressure

= free stream dynamic pressure

= steady pitching velocity (Fig. 2c)

= locus of surface of body of revolution

= reference area

= cross-sectional area of body of revolution at station $\mathrm{x}$

$=$ time in seconds

= free stream speed

= local velocity

= upwash velocity (Fig. I) 
$\mathrm{X}_{\mathrm{O}}$

$x, r, \theta$

$\mathrm{X}, \mathrm{Y}, \mathrm{Z}$

$\alpha$

$\beta=\sqrt{M^{2}-1}$

$\gamma$

$\phi_{0}$

$\phi, \phi_{1}, \phi_{2}$

$\psi$

$\omega$
UMM-80

= center of rotation of pitching motion

= cylindrical coordinates

= body stability axes through $\mathrm{x}_{\mathrm{O}}$

GREHEK SYMBOIS

= angle of attack

= compressibility parameter

= ratio of specific heats

= free stream velocity potential

= perturbation velocity potentials

= total velocity potential

= frequency of periodic motion 
UMM-80

\section{SUMMARY}

The general velocity potential for a slender pointed body of revolution oscillating periodically in a supersonic uniform stream is presented. The solution is shown to reduce in the limit to the sum of well-known stationary source-sink and doublet solutions when the frequency of oscillation approaches zero. Stability derivatives for low frequency oscillations are determined by making use of the approximate potential obtained by expanding the oscillating velocity potential in powers of reduced frequency, retaining only the first order terms in reduced frequency. In particular, the following four cases of steady and non-steady supersonic motion are treated: (1) harmonic pitching about a point $\mathrm{x}_{0}$; (2) harmonic normal oscillations in pitch; (3) steady pitching about a point $\mathrm{x}_{\mathrm{O}}$; and (4) steady angle of attack. Equations for the slender body theory normal force and pitching moment stability derivatives are also determined.

Two types of bodies of revolution are examined to demonstrate how the aerodynamic stability derivatives may be varied by changing the contour of the body. "Flaring" the aft end of the body increases the lift as well as increasing the aerodynamic damping moment of a finless body. 
UMM-80

\section{INTRODUCTION}

Attention has been directed lately to the problem of dynamic stability of low-aspect-ratio high-speed aircraft (Ref. 1 and 2). The problem of determining the static and dynamic aerodynamic force and moment stability derivatives of low-aspect-ratio wings at supersonic speeds has been treated by many investigators (Ref. 3, 4, 5, 6, 7, 8, and 9). Little consideration beyond first approximations has been given to the corresponding problems for bodies of revolution (Ref. 10). The dynamic stability derivatives for such bodies become of prime importance for finless missiles and missile configurations having relatively low area, low-aspect-ratio surfaces.

It is the purpose of this paper to present the general potential for the harmonic motion of a slender pointed body of revolution in a steady supersonic stream. Following this, the doublet potential for low frequency harmonic oscillations is demonstrated. The slender body theory doublet potentials are also determined. Example solutions for a body of revolution are calculated to illustrate the variation of the stability derivatives with Mach number in a limited supersonic Mach number range. It is shown how the stability derivatives can be modified considerably by varying the contour of the body of revolution.

The solutions for steady pitching and steady angle of attack are also presented for comparison purposes. 


\section{FUNDAMENTAL SOLUTIONS}

The well-known non-linear potential equation for compressible non-steady flow in cylindrical coordinates is (Ref. 11); figure 1,

$$
\Psi_{x x}+\frac{1}{\pi} \Psi_{\pi}+\Psi_{\pi r}+\frac{1}{\pi^{2}} \Psi_{\theta \theta}-\frac{1}{c^{2}}\left\{\Psi_{t t}+\frac{\partial V^{2}}{\partial t}+\vec{V} \overrightarrow{\operatorname{grad}} \frac{V^{2}}{2}\right\}=0
$$

Where, from the time dependent energy equation for compressible flow,

$$
\Psi_{t}+\frac{V^{2}}{2}+\frac{c^{2}}{\gamma^{2}-1}=F(t)
$$

We will proceed to linearize equation (1) in the customary manner by assuming that the desired solution to equation (I) can be represented by

$$
\psi=\varphi_{0}(x, \pi, \theta)+\varphi(x, \hbar, \theta, t)
$$

where

$$
\begin{array}{ll}
\varphi_{0}(x, \pi, \theta) & \text { is the free stream potential } \\
\varphi(x, h, \theta, t) & \text { is the perturbation potential represent- } \\
& \text { ing the unsteady body of revolution } \\
& \text { (considered small with respect to } \left.\phi_{0}\right)
\end{array}
$$

Should equation (3) be substituted into equations (1) and (2) and the products and second and higher powers of the derivatives of $\phi$ be neglected as being of secondary order of magnitude, we arrive at the Prandtl-Glauert equation for non-steady compressible flow.

$$
B^{2} \varphi_{x x}-\varphi_{r r-\frac{1}{n^{2}}} \varphi_{\theta \theta}-\frac{1}{r} \varphi_{r}+\frac{1}{c^{2}} \varphi_{t t}+\frac{2 M}{c} \varphi_{x t}=0
$$

The solutions with which this paper is concerned will satisfy equation (4). As such, these solutions are all subject to the usual limitations of the linearized theory. The solution sought in this 
WILLOW RUN RESEARCH CENTER UNIVERSITY OF MICHIGAN UMM-80

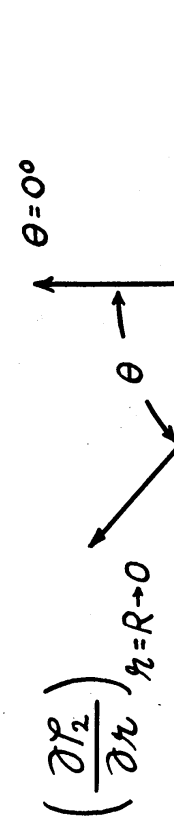

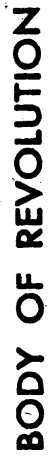

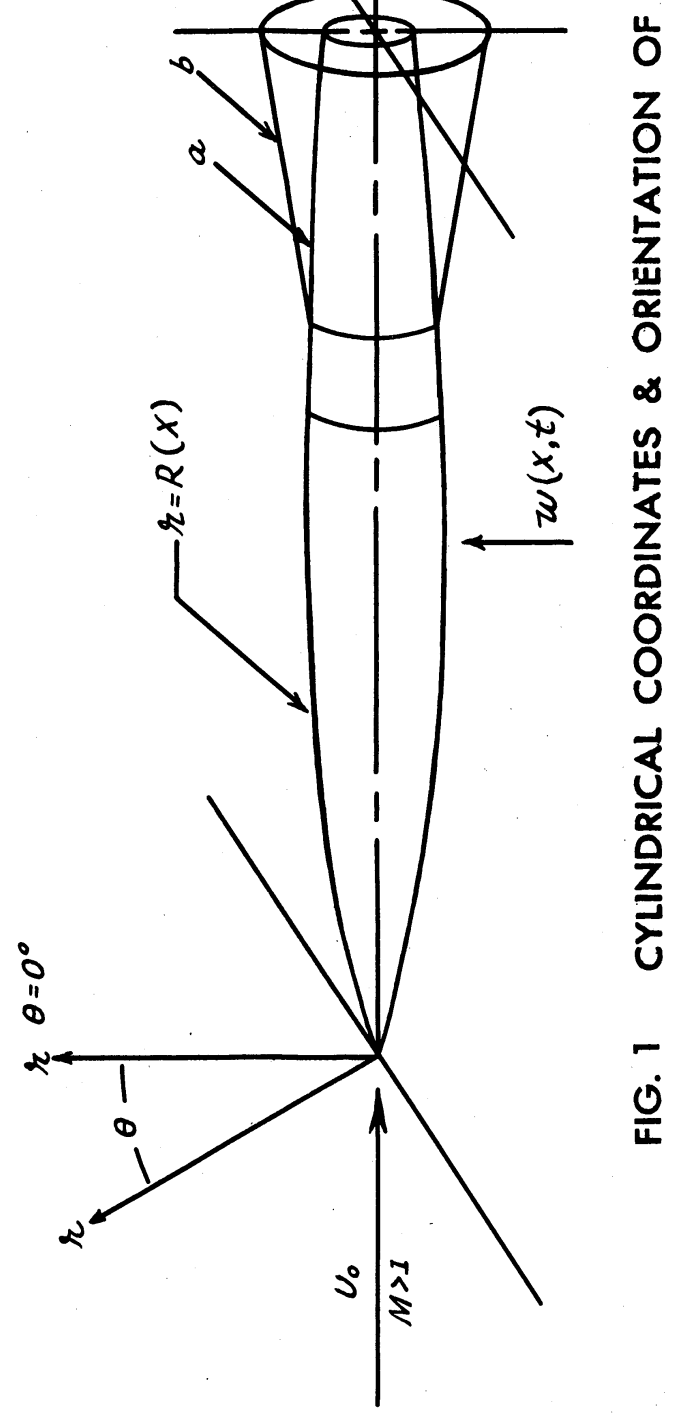


paper is

$$
\varphi_{2}=\frac{\partial \varphi_{1}}{\partial r} \cos \theta
$$

where $\phi_{I}$ is the solution to the equation

$$
B^{2} \varphi_{x x}-\varphi_{r r}-\frac{1}{r} \varphi_{n}+\frac{1}{c^{2}} \varphi_{t t}+\frac{2 M}{c} \varphi_{x t}=0
$$

Direct substitution of equation (5) into equation (4) will verify this. Here, the perturbation potential representing the body is composed of

$$
\varphi=\varphi_{1}+\varphi_{2}
$$

The potential $\phi_{1}$ is axially symmetric with respect to the $x$-axis and cannot yield "lifting" velocity perturbations (except as a product with the $\phi_{2}$ potential velocity perturbations). The potential $\phi_{2}$ being odd with respect to the plane $\theta=\frac{\pi}{2}$ will yield the desired lifting velocity perturbations.

\section{A. The Non-steady Source-sink Potential}

Let

$$
\varphi_{1}=-N(x, r) \exp \left\{i \omega\left(t-\frac{M x}{C B^{2}}\right)\right\}
$$

in equation (6). There results

$$
N_{\pi \Omega}+\frac{1}{\pi} N_{r}-B^{2} N_{x} x-\frac{\omega^{2}}{c^{2} B^{4}} N=0
$$

Further, make use of the Laplace transformation, and let 


$$
\overline{N(S, n)}=\int_{0}^{\infty} \exp \{-S x\} N(x, r) d x
$$

If $N(0, h)=\frac{\partial N}{\partial x}(0, h) \equiv 0$ appropriate to pointed bodies, operating upon equation (9) with equation (10) yields,

$$
\frac{d^{2} \bar{N}}{d r^{2}}+\frac{1}{r} \frac{d \bar{N}}{d r}-B^{2}\left(s^{2}+\frac{w^{2}}{c^{2} B^{4}}\right) \bar{N}=0
$$

The desired solution to equation (11) is the modified Bessel function of the second kind.

$$
\bar{N}(s, r)=K_{0}\left(B r\left[s^{2}+\frac{\omega^{2}}{C^{2} B^{4}}\right]^{1 / 2}\right)
$$

Using transform pair (871.5) of reference 12, we obtain

$$
N(x r)=\frac{\cos \left[\frac{\omega}{c B^{2}}\left(x^{2}-B^{2} r^{2}\right)^{\frac{1}{2}}\right]}{\left[x^{2}-B^{2} r^{2}\right]^{1 / 2}} \quad x>B r
$$

The point source solution to equation (6) is obtained through equations (8) and (13).

$$
\varphi_{1}=\frac{\exp \left\{i \omega\left(t-\frac{M x}{C B^{2}}\right)\right\} \cos \left[\frac{\omega}{C B^{2}}\left(x^{2}-B^{2} r^{2}\right)^{\frac{1}{2}}\right]}{\left[x^{2}-B^{2} r^{2}\right]^{1 / 2}} x>B r
$$

If the sources are distributed along the $\mathrm{x}$-axis at points $\xi$ according to $f(\xi)$, then equation (14) becomes

$$
\begin{gathered}
\varphi_{1}=-\exp \{i \omega t\} \int_{0}^{x(\xi) \exp \left\{-\frac{i \omega M}{c B^{2}}(x-\xi)\right\} \cos \left(\frac{\omega}{C B^{2}}\left[(x-\xi)^{2}-B^{2} r^{2}\right]^{1 / 2}\right) d \xi} \\
{\left[(x-\xi)^{2}-B^{2} r^{2}\right]^{1 / 2}} \\
(x-\xi)>B r
\end{gathered}
$$


if $\omega=0$, as in the steady source-sink solution, then equation

(15) becomes the easily recognized form

$$
\begin{aligned}
\varphi_{1}=-\int_{0}^{x-B r} \frac{f(\xi) d \xi}{\left[(x-\xi)^{2}-B^{2} r^{2}\right]^{\frac{1}{2}}} \\
x-\xi>B r
\end{aligned}
$$

B. The Non-steady "Doublet" Potential

Equations (5) and (15) are now employed to obtain the desired supersonic non-steady doublet potential. The differentiation indicated in equation (5) involves differentiating under the integral sign of an improper integral. This differentiation is presented in Appendix $A$ and results in the doublet potential below.

$\varphi_{2}=\exp \{i \omega t\} \cos \theta\left\{\frac{\omega}{r C B^{2}} \int_{0}^{x-B r} f(\xi) \exp \left\{\frac{-i \omega M}{C B^{2}}(x-\xi)\right\} \sin \left(\frac{\omega}{C B^{2}}\left[(x-\xi)^{2}-B^{2} r^{2}\right]^{\frac{1}{2}}\right) d \xi\right.$

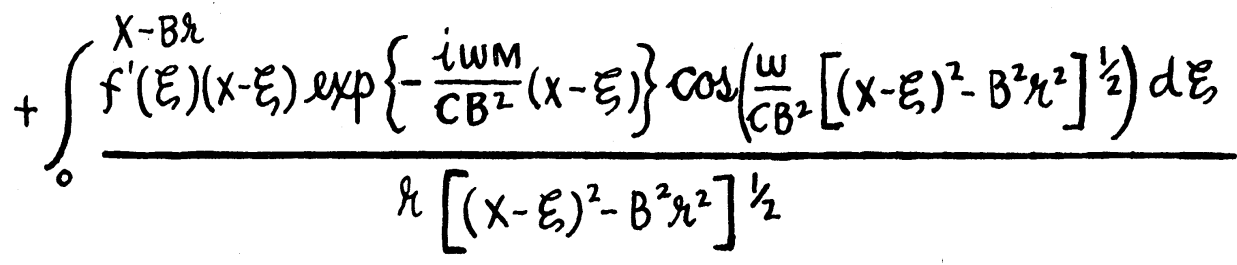

$$
+\frac{i \omega M}{C B^{2} r} \int_{0}^{\frac{x-B r}{f(\xi)(x-\xi) \exp \left\{-\frac{i \omega M}{C B^{2}}(x-\xi)\right\} \cos \left(\frac{\omega}{C B^{2}}\left[(x-\xi)^{2}-B^{2} r^{2}\right]^{\frac{1}{2}}\right) d \xi}}
$$

If, in equation (17), $\omega=0$, the recognized steady doublet potential results.

$$
\varphi_{2}=\frac{\cos \theta}{r} \int_{0}^{\frac{x-B r}{f^{\prime}(\xi)(x-\xi) d \xi}} \frac{x-\xi>B r}{\left[(x-\xi)^{2}-B^{2} r^{2}\right]^{\frac{1}{2}}} \quad x-\xi
$$


Equation (17) is the desired non-steady doublet solution to equation (4). The balance of this paper will be devoted to presenting particular solutions to equation (4) appropriate to: (a) harmonic pitching about a point $\mathrm{x}_{0}$; (b) harmonic normal oscillations in pitch; (c) steady pitching about a point $\mathrm{x}_{\mathrm{O}}$; and (d) steady angle of attack. All of these situations can be treated by making use of equation (17) and its derivatives.

C. Boundary Conditions

The boundary conditions for the steady or non-steady supersonic flow about a body of revolution specify that the velocity component normal to the surface of the body at the surface must be zero. That is, if

$$
h=R(x) \quad 0 \leqq x \leqq L
$$

is the locus of the surface of the body in all meridian planes, then the boundary condition is (Fig. 1)

$$
\left(\frac{\partial \varphi_{2}}{\partial r}\right)_{R \rightarrow 0}=-w(x, t) \cos \theta
$$

in general. Equation (20) takes a different form for each of the four steady and non-steady motions previously mentioned.

1. Harmonic pitching about the point $x_{0}$

In this case $\alpha(t)=\alpha_{0} \exp \{i \omega t\}$, and from figure $2 a$ and equation (20)

$$
\left(\frac{\partial \varphi_{2}}{\partial r}\right)_{R \rightarrow 0}=-\alpha_{0} \exp \{i \omega t\} \cos \theta\left[U_{0}+i \omega\left(x-x_{0}\right)\right]
$$


WILLOW RUN RESEARCH CENTER UNIVERSITY OF MICHIGAN UMM-80

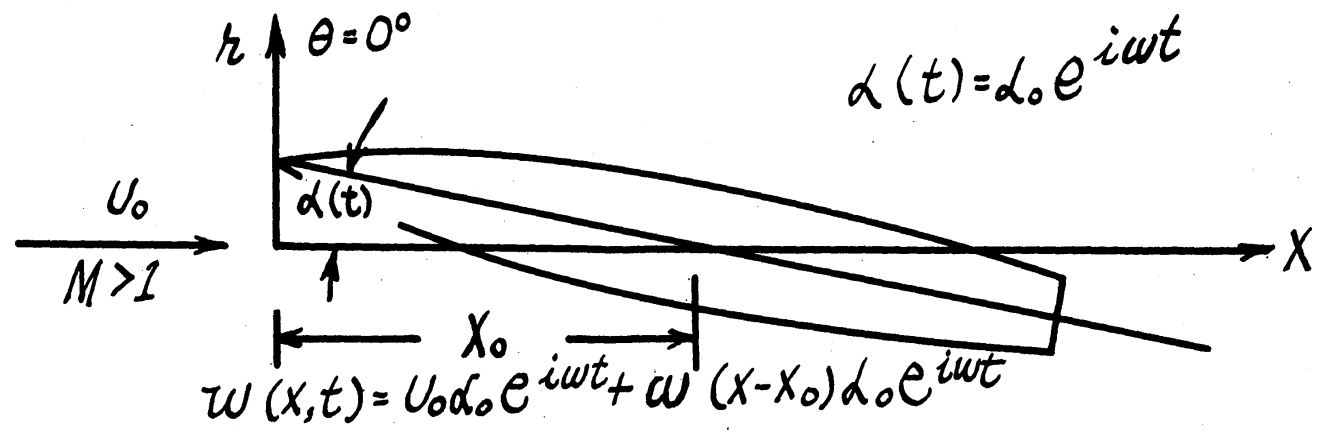

FIG. 2a HARMONIC PITCHING ABOUT XO

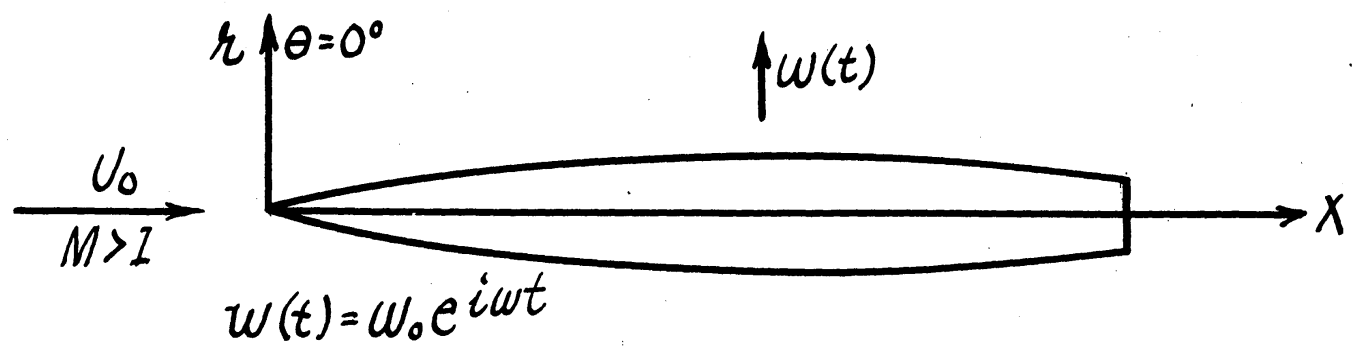

FIG. $2 b$ HARMONIC NORMAL OSCILLATIONS IN PITCH

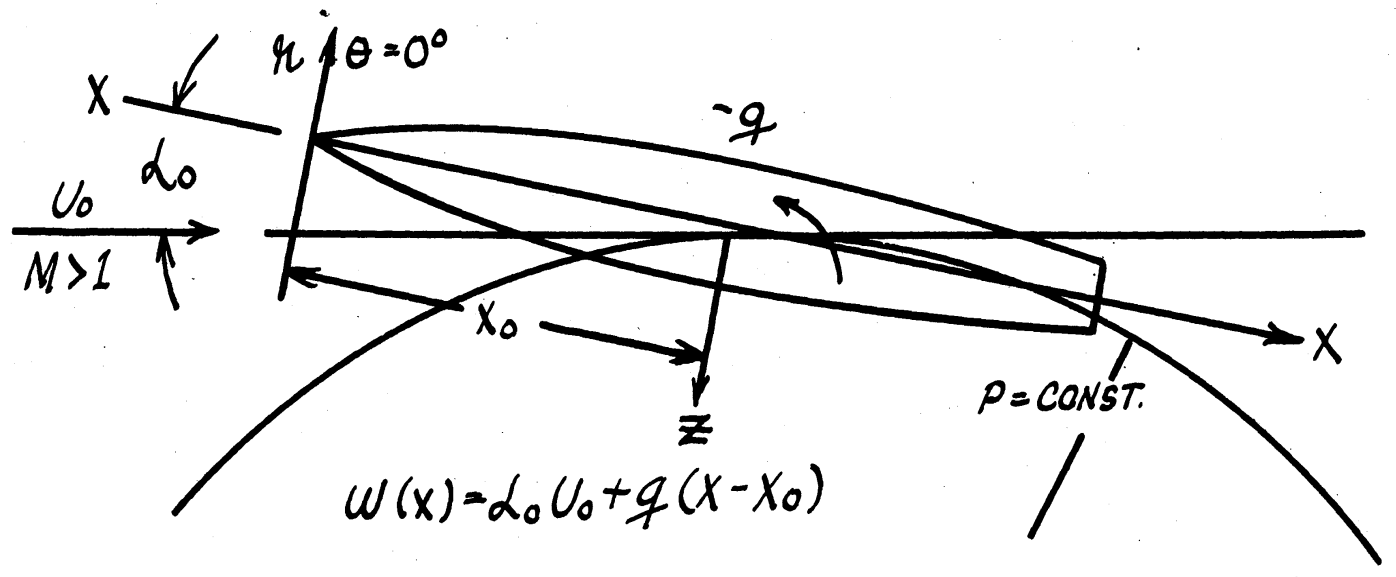

FIG. 2c STEADY PITCHING ABOUT $X_{O}$

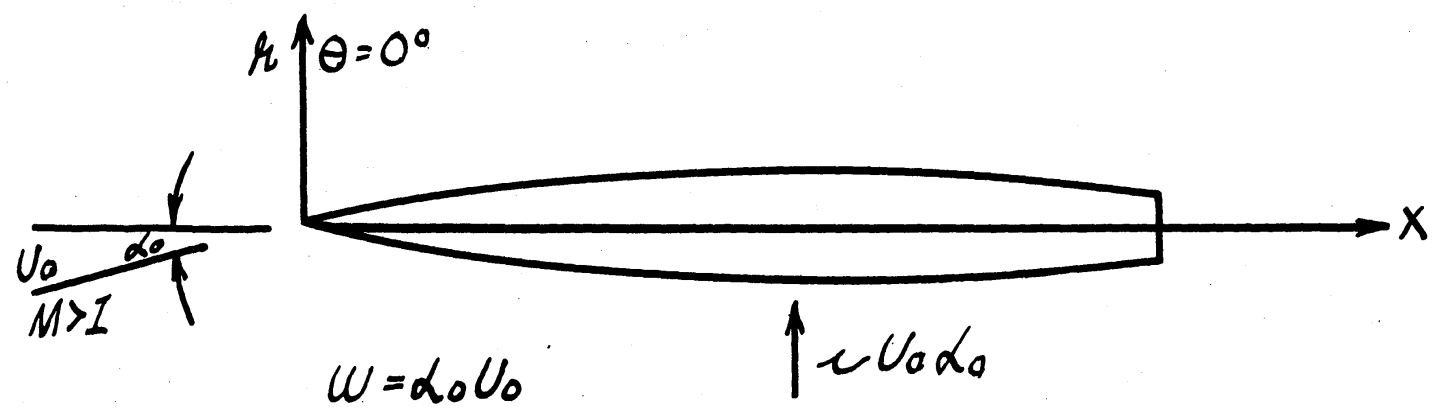

FIG. 2d STEADY ANGLE OF ATTACK 
2. Harmonic normal oscillations in pitch

$$
\left(\frac{\partial \varphi_{2}}{\partial r}\right)_{R \rightarrow 0}=\omega_{0} \exp \{i \omega t\} \cos \theta
$$

3. Steady pitching about the point $\mathrm{x}_{0}$

From figure $2 c$ and equation (20)

$$
\left(\frac{\partial \varphi_{2}}{\partial r}\right)_{R \rightarrow 0}=-\cos \theta\left[U_{0} \alpha+q\left(x-x_{0}\right)\right]
$$

The capital letters $\mathbf{X}$ and $\boldsymbol{Z}$ in figure $2 c$ denote body stability axes. The point $\mathrm{x}_{\mathrm{O}}$ is the center of gravity, and the steady pitching velocity is taken in its defined sense.

4. Steady angle of attack

From figure $2 d$ and equation (20)

$$
\left(\frac{\partial \varphi_{2}}{\partial r}\right)_{R \rightarrow 0}=U_{0} \alpha \cos \theta
$$

This case has been treated in the literature before (Ref. 13 and 14) and will not be dwelt upon in any great detail in this paper, other than to compare it with the other solutions.

Equations (17) and (18), together with equations (21), are now used to specify the function $f(\xi)$. The value of $\left(\frac{\partial \phi_{2}}{\partial r}\right)_{R \rightarrow 0}$ is obtained in Appendix $B$, where it is shown that

$$
\left(\frac{\partial \varphi_{2}}{\partial r}\right)_{R \rightarrow 0}=\frac{f(x)}{R^{2}} \exp \{i \omega t\} \cos \theta
$$

The function $f(\xi)$ is now determined through equations (21) and (22) for all of the cases treated in this paper. 
The steady and non-steady pressure coefficients can be obtained from the general Bernoulli equation.

$$
\psi_{t}+\frac{v^{2}}{2}+\int_{p_{T}}^{p} \frac{d p}{p}=F(t)
$$

Equation (3) is substituted into the Bernoulli equation, and terms are dropped in keeping with the process used to linearize equation (1). Keeping only terms odd with respect to the plane $\theta=\frac{\pi}{2}$ results in the approximate "lifting" pressure coefficient.

$$
c p=-2\left\{\frac{\varphi_{2} x}{U_{0}}+\frac{\varphi_{2} t}{U_{0}^{2}}\right\}
$$

If normal force coefficient and moment coefficient are defined as

$$
C_{n}=-\frac{2}{s} \int_{0}^{L} \int_{0}^{\pi} c p R \cos \theta d \theta d x
$$

and

$$
C_{m}=\frac{-2}{S L} \int_{0}^{L} \int_{0}^{\pi} C p R\left(\chi_{0}-\psi\right) \cos \theta d \theta d \psi
$$

because $C_{p}$ is proportionate to $\cos \theta$, it follows that

$$
c_{n}=-\frac{\pi}{s} \int_{0}^{L}\left[\frac{c p}{\cos \theta}\right] R d x
$$


and

$$
C_{m}=C_{n} \frac{x_{0}}{L}+\frac{\pi}{s L} \int_{0}^{L}\left[\frac{o p}{\cos \theta}\right] R x d x
$$

We are now in a position to obtain the lift and pitching moment coefficients for the four motions discussed in Section IV. 
The task of obtaining solutions for the non-steady supersonic flow about bodies of revolution using equation (17) is simplified for the case of low frequency oscillations. It can be shown by expanding equation (17) into powers of reduced frequency that for harmonic frequencies of the order of $\frac{C B}{I}$ the non-steady potential can be represented by the first order terms in $\mathrm{k}$ with an error of about $5 \%$ or less. For bodies of the order of 20 to 30 feet in length in the Mach number range of 1.5 to 2.5 , the first order terms in $k$ in equation (17) would represent the non-steady potential for harmonic frequencies of the order of 35 to 115 radians per second within about 5\%. Before proceeding to this expansion process circumvent the improper integrals in equation (17) by introducing the dummy variable $\mu$ through the substitution

$$
\cosh u=\frac{x-\xi}{B r}
$$

If equation (26) is substituted into equation (17), there results

$$
\varphi_{2}=-\cos \theta \exp \{i \omega t)\left\{B \int_{\cosh ^{-1} \frac{\psi}{B r}} \int^{0}(\xi) \exp \left\{\frac{-i \omega M r}{C_{B}} \cosh u\right\} \cos \left(\frac{\omega r}{C_{B}} \sinh u\right) \cosh u d u\right.
$$

$$
+\frac{\omega}{C B} \int_{\cosh ^{-1} \frac{\psi}{B r}}^{0} f(\xi) \exp \left\{\frac{-i \omega M r}{C B} \cosh u\right\} \sin \left(\frac{\omega r}{C B} \sinh u\right) \sinh u d u
$$

$$
\left.+\frac{i \omega M}{C B} \int_{\cosh ^{-1} \frac{x}{B r}}^{0} f(\xi) \exp \left\{\frac{-i \omega M r}{C B} \cosh u\right\} \cos \left(\frac{\omega r}{C B} \sinh u\right) \cosh u d u\right\}
$$


carrying out the expansion into powers of $\mathrm{k}$ we obtain

$\varphi_{2}=-\cos \theta \exp \{i \omega t\}\left\{B \int_{\cosh ^{-1} \frac{\psi}{B r}}^{0} f^{\prime}(x-B r \cosh u) \cosh u d u\right.$

$$
\left.-i M B k \int_{\cosh ^{-1} \frac{x}{B r}}^{0} f^{\prime}(x-B r \cosh u) d u+0\left(k^{2}\right)\right\}
$$

If $\omega=0$ in equation (28), the well-known steady potential results.

$$
\varphi_{2}=\cos \theta B \int_{\cosh ^{-1} \frac{\psi}{B r}}^{0} f^{\prime}(x-B r \cosh u) \cosh u d u
$$

Equations (28) and (29) will be used to obtain the four non-steady and steady solutions with which we are concerned.

\section{A. Harmonic Pitching About the Point $x_{0}$}

The equation for the distribution $f(\xi)$ is obtained from equations (2la) and (22).

$$
f(\xi)=\frac{s(\xi)}{\pi}\left[U_{0} \alpha_{0}+i \omega\left(x-x_{0}\right) \alpha_{0}\right]
$$

Equation (30) is substituted into equation (28) and equation (28) substituted into equation (23) to obtain the pressure coefficient on the body.

$$
\begin{gathered}
C_{p}=\frac{2 \cos \theta}{\pi^{i}}\left\{\left(\alpha B-\frac{B^{2} \alpha x_{0}}{U_{0}}\right) \frac{\partial I_{1}}{\partial x}+\frac{\alpha B}{U_{0}} \frac{\partial I_{2}}{\partial y}+2 \frac{\alpha B}{U_{0}} I_{1}\right. \\
\left.-\frac{\dot{\alpha} M^{2} R}{U_{0}} \frac{\partial I_{4}}{\partial y}+O\left(k^{2}\right)\right\}
\end{gathered}
$$


The integrals $I_{n}^{\prime}$ appearing in equation (3I) are presented in Appendix C. These integrals will also appear in the remaining expressions for the steady and non-steady pressure coefficients to be determined. Equation (31) is now substituted into equations (24) and (25) to obtain the desired derivatives.

$$
C_{n_{\alpha}}=-\frac{2}{S L} \int_{0}^{L}\left[2 B\left(I_{1}+\frac{1}{2} \frac{\partial I_{2}}{\partial x}\right)-M^{2} R \frac{\partial I_{4}}{\partial x}-B x_{0} \frac{\partial I_{1}}{\partial x}\right] R d \psi
$$

$$
C_{m_{2}}=C_{n_{2}} \frac{\psi_{0}}{L}+\frac{2}{S L^{2}} \int_{0}^{L}\left[2 B\left(I_{1}+\frac{1}{2} \frac{\partial I_{2}}{\partial x}\right)-M^{2} R \frac{\partial I_{4}}{\partial \psi}-B x_{0} \frac{\partial I_{1}}{\partial \psi}\right] R \psi d \psi
$$

The slender body theory expression for pressure coefficient is obtained by substituting equation (2la) into equation (B-6) in Appendix. $B$ and substituting the resulting equation into equation (23). This equation is then substituted into equations (24) and (25) to obtain the following:

$$
C_{n_{\alpha}}=2 \frac{S(L)}{S}\left[\left(1-\frac{\psi_{0}}{L}\right)+\frac{V O L}{L S(L)}\right]
$$

and

$$
C_{m_{\alpha}}=-2 \frac{s(L)}{s}\left(1-\frac{x_{0}}{L}\right)^{2}
$$

B. Harmonic Normal Oscillations in Pitch

From equations (21b) and (22), we have

$$
f(\xi)=\frac{s(\xi)}{\pi} \omega_{0}
$$




\section{WILLOW RUN RESEARCH CENTER - UNIVERSITY OF MICHIGAN}

UMM-80

Equation (36) is used in equations (28), (23), (24), and (25), respectively to yield

$$
C_{\eta_{\dot{\omega}}}=\frac{-2}{S L} \int_{0}^{L}\left[B I_{1}-M^{2} R \frac{\partial I_{4}}{\partial \psi}\right] R d \psi
$$

and

$$
C_{m_{\dot{\omega}}}=C_{n_{\dot{\omega}}} \frac{\chi_{0}}{L}+\frac{2}{S L^{2}} \int_{0}^{L}\left[B I_{1}-M^{2} R \frac{\partial I_{4}}{\partial \psi}\right] R \psi d \psi
$$

The corresponding slender body theory expressions are:

$$
C_{n_{\dot{\omega}}}=2 \frac{\text { VOL. }}{S L}
$$

and

$$
C_{m_{\dot{\omega}}}=2 \frac{S(L)}{S}\left[\frac{\psi_{0}}{L} \frac{V_{0} L}{S(L) L}-\int_{0}^{L} \frac{S(x) \psi d \psi}{S(L) L^{2}}\right]
$$

C. Steady Pitching About the Point

Equations (2lc) and (23) yieldd

$$
f(\xi)=\frac{s(\xi)}{\pi} q\left(\xi-\psi_{0}\right)
$$


Substitution of equation (41) into equation (29) and the resulting equation into equation (23) and thence to equations (24) and (25) gives

$$
C_{\eta_{q}}=\frac{-2 B}{S L} \int_{0}^{L}\left[I_{1}+\frac{\partial I_{2}}{\partial x}-x_{0} \frac{\partial I_{1}}{\partial y}\right] R d y
$$

and

$C_{m q}=C n_{q} \frac{x_{0}}{L}+\frac{\partial B}{S L^{2}} \int_{0}^{L}\left[I_{1}+\frac{\partial I_{2}}{\partial \psi}-x_{0} \frac{\partial I_{1}}{\partial \psi}\right] n R d \psi$

The corresponding slender body theory expressions are:

$$
C_{n_{q}}=2 \frac{S(L)}{S} 1-\frac{\psi_{0}}{L}
$$

and

$$
c_{m g}=-2 \frac{S(L)}{S}\left(1-\frac{x_{0}}{L}\right)^{2}+\frac{x_{0}}{L} \frac{V_{0 L}}{L S(L)}-\frac{\int_{0}^{L} S(x) \psi d \psi}{L^{2} S(L)}
$$

D. Steady Angle of Attack

From equations (2ld) and (23)

$$
f(\xi)=\frac{s(\xi)}{\pi} U_{0} \alpha
$$


Then, from equations (46), (29), (23), (24), and (25)

$$
\begin{gathered}
C_{n \alpha}=\frac{-2 B}{S} \int_{0}^{L}\left[\frac{\partial I_{1}}{\partial \psi}\right] R d \psi \\
C_{m_{\alpha}}=C_{n_{\alpha}} \frac{x_{0}}{L}+\frac{2 B}{S L} \int_{0}^{L}\left[\frac{\partial I_{1}}{\partial \psi}\right] R \psi d \psi
\end{gathered}
$$

The slender body theory results are:

$$
c_{n_{\alpha}}=2 \frac{S(L)}{S}
$$

and

$$
C_{\text {m } \alpha}=2 \frac{S(L)}{S}\left(\frac{X_{0}}{L}+\frac{V_{0 L}}{L S(L)}-1\right)
$$

This completes the list of steady and non-steady stability derivatives. These equations will be demonstrated in the next section for two different bodies of revolution. 
UMM-80

\section{EXAMPIE RESULTS FOR TWO BODIES OF REVOLUTION}

The equations obtained in the previous section will now be applied to the two bodies shown in figure 1. The stability derivatives for body (a) of figure 1 will be obtained using both the first order in frequency equations and the slender body theory. Following this, it will be shown how modifying the shape of body (a) to body ( $b$ ) changes the stability coefficients.

Body (a) of figure $I$ is generated by revolving a parabolic arc about the x-axis. The equation of this parabolic arc is

$$
R=.02\left(10 x-x^{2}\right) \quad 0 \leqq x \leqq 8
$$

Body (b) incorporates the nose of body (a) with a short cylindrical section and a slightly "flaring" rear section. The equation for the locus of the surface of body (b) is

$$
\begin{array}{ll}
R=.02\left(10 x-x^{2}\right) & 0 \leqq x \leqq 5 \\
R=.50 & 5 \leqq x \leqq 6 \\
R=.08715 x-.0229 & 6 \leqq x \leqq 8
\end{array}
$$

The point of rotation $x_{0}$ for the steady and non-steady pitching derivative was taken to be $x_{0}=4.78$ for both bodies. This point was chosen as being typical for certain rocket-propelled missiles.

Figure 3 presents the values of $\mathrm{C}_{n_{\alpha}}$ and $\mathrm{C}_{\mathrm{m}_{\dot{\alpha}}}$ for body (a) using equations (32), (33), (34), and (35). The limitations of the slender body theory in predicting the magnitude and variation with Mach number of these stability coefficients is apparent. This depends, of course, on the validity of the first order in frequency theory. 


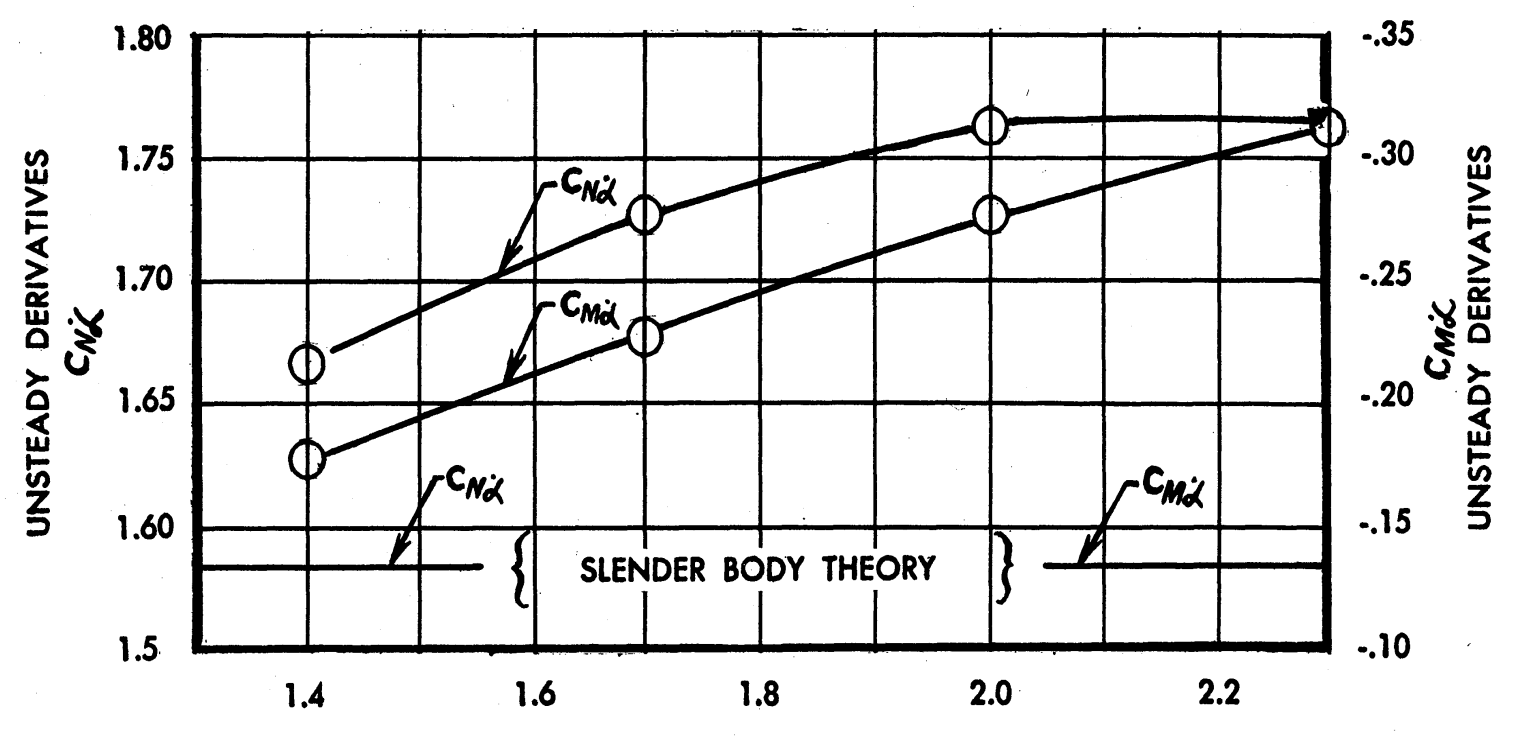

MACH NUMBER

FIG. 3 VALUES OF CNd AND CMd FOR BODY (a)

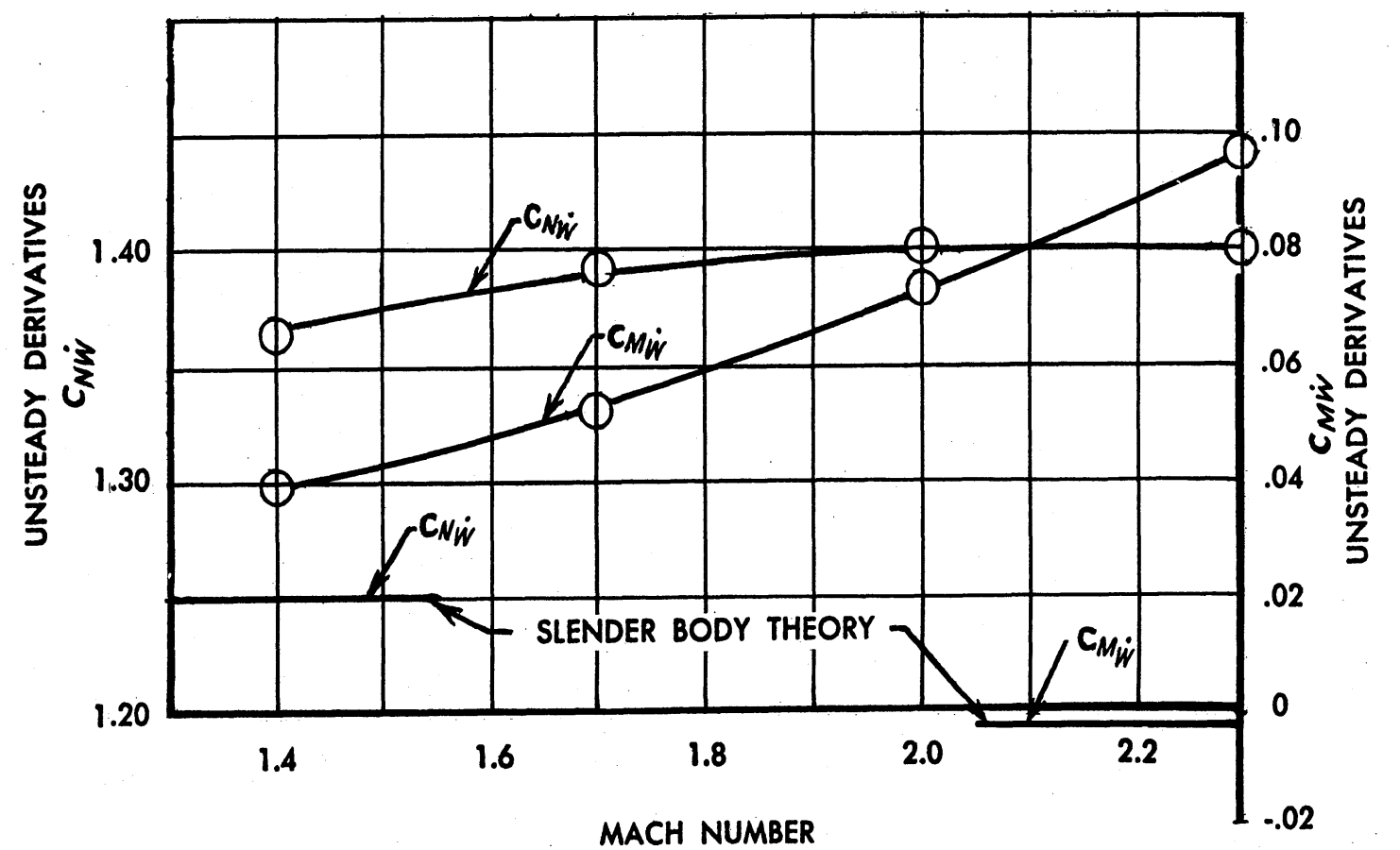

Fig. 4 VALUES OF $C_{N} \dot{W}, C_{M} \dot{W}$ FOR BODY (a) 
Figures 4, 5, and 6 present values of $\mathrm{C}_{\mathrm{n}_{\dot{\mathrm{w}}}}, \mathrm{C}_{\mathrm{m}_{\dot{\mathrm{w}}}}, \mathrm{C}_{\mathrm{n}_{\alpha}}, \mathrm{C}_{\mathrm{m}_{\alpha}}, \mathrm{C}_{\mathrm{n}_{\mathrm{q}}}$, and $\mathrm{C}_{\mathrm{m}_{\mathrm{q}}}$ for body (a) using both theories. The remarks above relative to the two theories apply to these results also.

An interesting result of these calculations is obtained by comparing figures 3 and 6 which present the non-steady and steady pitching derivatives, respectively. In dynamic stability analyses the steady pitching derivatives $\mathrm{C}_{\mathrm{n}_{\mathrm{q}}}$ and $\mathrm{C}_{\mathrm{m}_{\mathrm{q}}}$ are often used rather than the non-steady derivatives $\mathrm{C}_{\mathrm{n}_{\dot{\alpha}}}$ and $\mathrm{C}_{\mathrm{m}_{\dot{\alpha}}}$. If this procedure were followed for body (a), both the magnitudes of the derivatives and the variation of the pitching moment derivative with Mach number would be in error in the Mach number range of figures 3 and 6 . In both cases, however, the sign of the pitching moment derivative is such as to indicate a damping moment due to a pitching angular velocity.

The slender , body theory derivatives for both bodies are given in Table 1 , and indicate qualitatively the effects of changing the shape of body (a) to that of body (b). All coefficients are based on the same reference area; the cross-sectional area at $\mathrm{x}=5$.

$\underline{\text { TABEE } 1}$

Slender Body Theory Stability Derivatives

$\begin{array}{lrrrrrrrr}\text { body } & C_{n_{\alpha}} & { } m_{\alpha} & C_{n_{q}} & C_{m_{q}} & C_{n_{\alpha}} & C_{m_{\dot{\alpha}}} & C_{n_{\dot{w}}} & C_{m_{\dot{w}}} \\ \text { (a) } & .82 & .93 & .33 & -.13 & 1.58 & -.13 & 1.25 & -.002 \\ \text { (b) } & 3.63 & .15 & 1.47 & -.48 & 3.08 & -.59 & 1.61 & -.114\end{array}$

Table 1 shows that body (b) has increased lift over that of body (a). It is also apparent from the values of $\mathrm{C}_{\mathrm{m}_{\alpha}}$ that the static center of pressure has moved rearward with changing body (a) to body $(b)$. Thus, body (b) has improved margin of static stability over that of body (a).

Both bodies possess steady and non-steady damping in pitching about $\mathrm{x}_{\mathrm{O}}$. However, body (b) shows an increase in both $\mathrm{C}_{\mathrm{m}_{\mathrm{q}}}$ and $\mathrm{C}_{\mathrm{m}_{\dot{\alpha}}}$ 
WILLOW RUN RESEARCH CENTER - UNIVERSITY OF MICHIGAN UMM-80

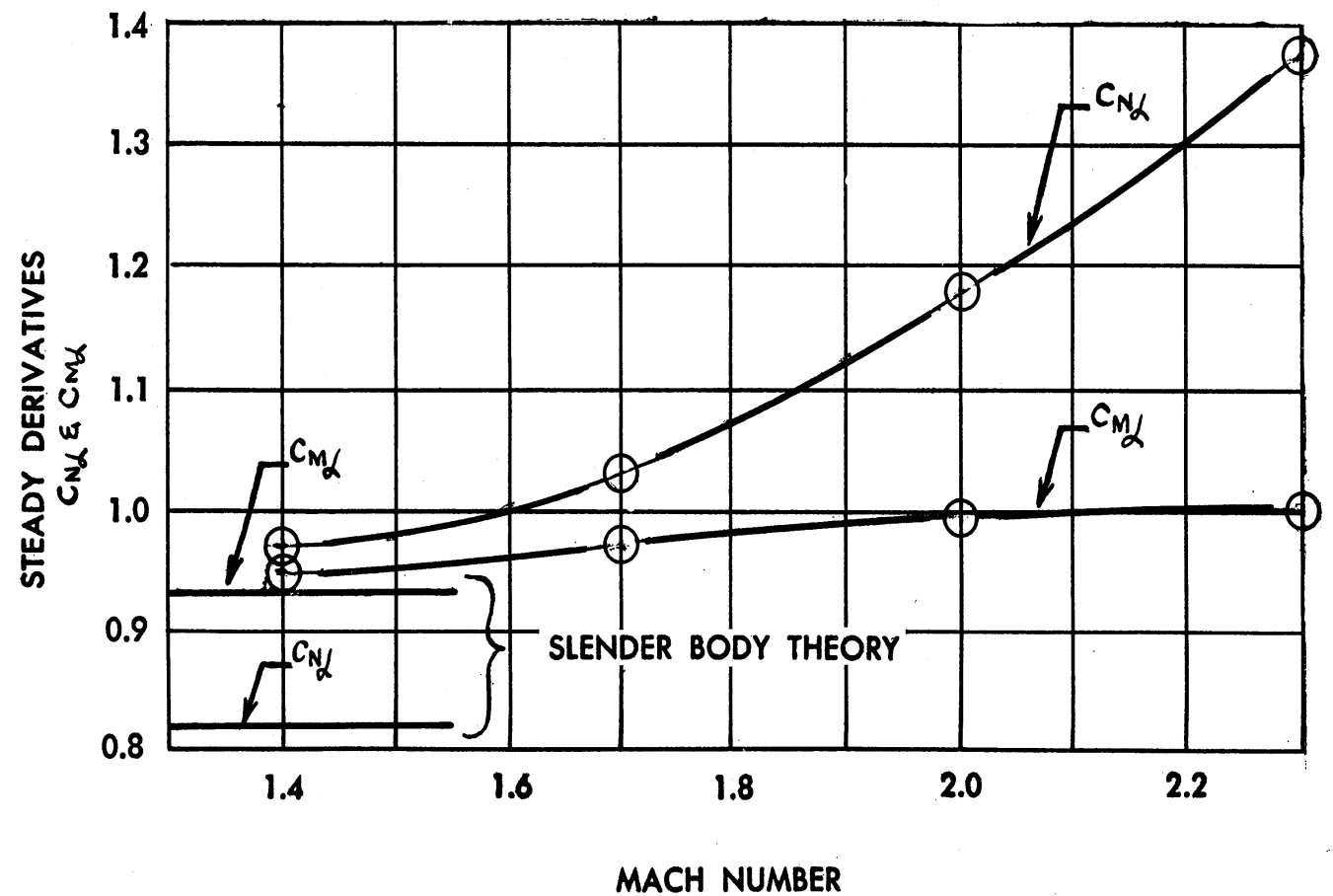

FIG. 5 VALUES OF $C_{N_{\alpha}}, C_{M} \alpha$ FOR BODY (a)

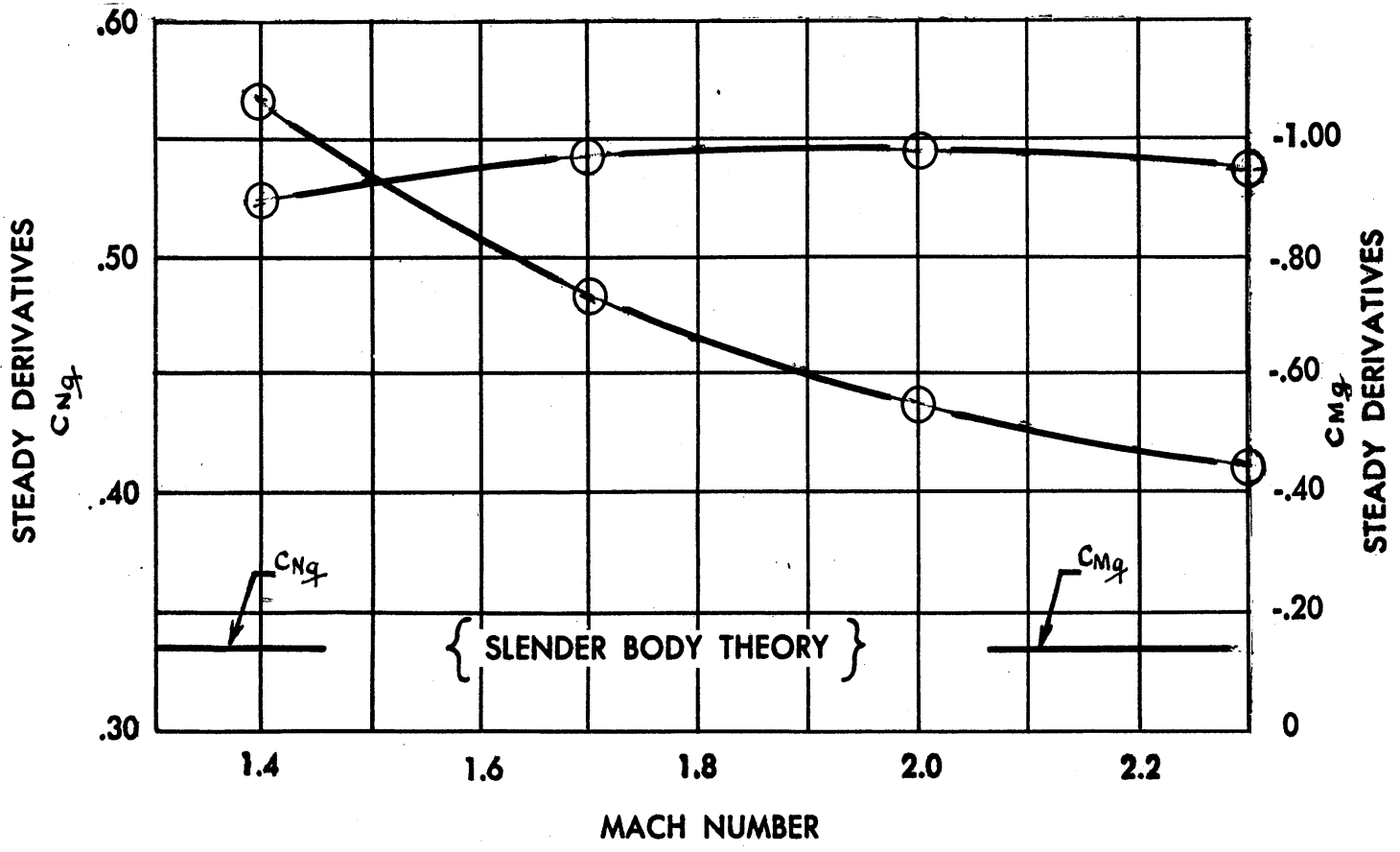

FIG. 6 VALUES OF $C_{N_{q}}$,AND $C_{M q}$ FOR BODY (a) 
over those values appropriate to body (a). Thus, body (b) has greater damping in pitch than body (a).

The above discussion indicates briefly how the aerodynamic properties of bodies of revolution can be modified by varying the shape of the body. In general, it can be stated that increasing the base area of a body of revolution will improve its steady and non-steady lift and pitching moment characteristics in the supersonic range of Mach numbers. 
Derivation of the Non-steady Supersonic Doublet Potential

Equations (5) and (15) can be combined to obtain the supersonic doublet potential for bodies of revolution. However, the differentiation demanded by equation (5) involves differentiating the improper integral in equation (15). The differentiation is simplified if the dummy variable is first introduced into equation (15) through the substitution

$$
\cosh \mu=\frac{x-\xi}{B r}
$$

If equation (A-I) is substituted into equation (15), there results

$\varphi_{1}=\exp \{i \omega t\} \int_{\cosh \frac{-1 \psi}{B M}}^{0} f(\xi) \exp \left\{-\frac{i \omega M \mu}{C B} \cosh \mu\right\} \cos \left(\frac{\omega r}{C B} \operatorname{sink} \mu\right)$

The differentiation required in equation (5) is easily performed to give

$\varphi_{2}=-\cos \theta \exp \{i \omega t\}\left\{B \int_{\cosh \frac{-1 \psi}{B r}}^{f^{\prime}(\xi) \exp }\left\{\frac{-i \omega M r}{C B} \cosh u\right\} \cos \left(\frac{\omega r}{C B} \sin k \mu\right) \cosh u d u\right.$

$\frac{+\omega}{C B} \int_{\cosh -\frac{-1 \psi}{B}}^{0}(\xi) \exp \left\{\frac{-i \omega M \pi}{C B} \cosh \mu\right\} \sin \left(\frac{\omega \pi}{C B} \operatorname{sink} \mu\right) \operatorname{sink} \mu d u$

$\left.+\frac{i \omega M}{C B} \int_{\cosh \frac{-i \psi}{B \Omega}}^{0} f(\xi) \exp \left\{\frac{-i \omega M r}{C B} \cosh \mu\right\} \cos \left(\frac{\omega r}{C B} \operatorname{sink} u\right) \cosh u d u\right\}$ 
where $f(0)$ is taken to be zero for pointed bodies. Substitution of equation ( $A-1$ ) into equation (A-3) yields equation (17). 


\section{APPENDIX B}

$$
\text { Evaluating } \lim _{R \rightarrow 0}\left(\frac{\partial \varphi_{2}}{\partial r}\right) r=R
$$

From equation (20) we have

$$
\left(\frac{\partial \varphi_{2}}{\partial r}\right)_{R \rightarrow 0}=-\omega\left(x_{1} t\right) \cos \theta
$$

Now, from equation (27)

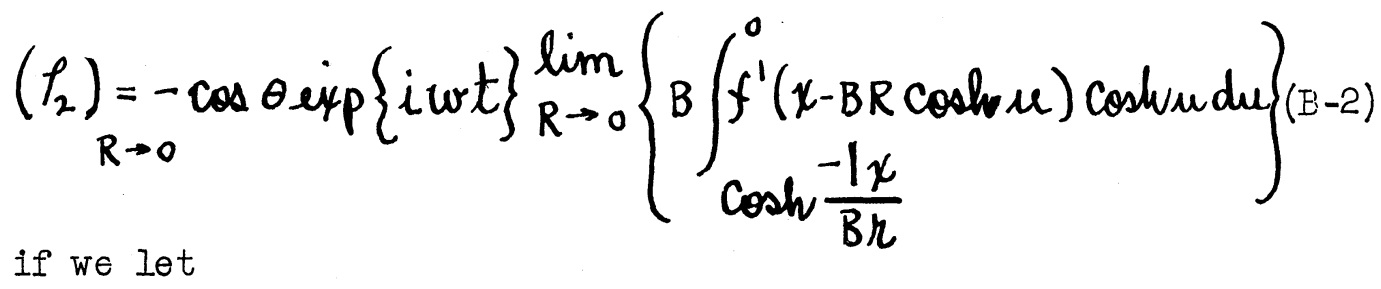

$$
\cosh \mu=\frac{\chi-\xi}{B R}
$$

Then equation ( $\mathrm{B}-2$ ) becomes

$\left(\varphi_{2}\right)=\cos \theta \exp \{i \omega t\} \lim _{R \rightarrow 0} 1 \int_{R \rightarrow 0}^{\psi} \frac{f^{\prime}(\xi)(\psi-\xi) d \xi}{\left[(\psi-\xi)^{2}-B^{2} R^{2}\right]^{\frac{1}{2}}}$

This gives

$$
\left(P_{2}\right)_{R \rightarrow 0}=\frac{f(x)}{R} \operatorname{sep}\{i \omega t\} \cos \theta
$$


and so

$$
\left(\frac{\partial \varphi_{2}}{\partial r}\right)_{R \rightarrow 0}=\frac{-f(x) \exp \{i \omega t\} \cos \theta}{R^{2}}
$$

The combination of equations $(B-1)$ and $(B-6)$ yields

$$
f(x)=\frac{s(x)}{\pi} w(x, t) \exp \{-i \omega t\}
$$

Equations (B-5) and (B-7) can be combined to yield the slender body theory non-steady potential for bodies of revolution.

$$
\varphi_{2}=\frac{S(x) \omega(x, t) \cos \theta}{\pi R}
$$


The Integrals $I_{n}(n=1,2,3$, 4) Appearing in the Steady and Non-steady Pressure Coefficients

Certain integrals appear in any or all of the expressions for pressure coefficient derived in this paper. These integrals are:

$$
\begin{aligned}
& I_{1}=\int_{\cosh \frac{-1 \psi}{B R}}^{0} S^{\prime}(x-B R \cosh u) \cosh u d u \\
& I_{2}=\int_{\cosh \frac{-1 x}{B R}}^{0} S^{\prime}(x-B R \cosh u)(x-B R \cosh u) \cosh u d u \\
& I_{3}=\int_{\cosh ^{-1 x}}^{0} S(x-B R \cosh u) \cosh u d u \\
& I_{4}=\int_{S^{\prime}(x-B R \cosh u) d u}^{\cosh \frac{-1 x}{B R}}
\end{aligned}
$$

When these integrals are differentiated with respect to $\mathrm{x}$, it should be remembered that $S(0)=S^{l}(0) \equiv 0$ for pointed bodies of revolution. 
UMM-80

No.

Title

1. Donovan, A. F., Flax, A. H., and Cheilek, H. A., "Stability and Control of Supersonic Aircraft", Institute of the Aeronautical Sciences Preprint No. 136, January 1948.

2. UMM-37 - University of Michigan, "The Stability and Response of an Aerodynamic Missile in Plane Motion", by H. G. Mazurkiewicz and R. M. Spath, December 1949.

3. Ribner, H. S., "The Stability Derivatives of Low-aspect-ratio Triangular Wings at Subsonic and Supersonic Speeds", N.A.C.A. T.N. No. 1423, 1947.

4. Ribner, H. S. and Malvestuto, F. S., Jr., "Stability Derivatives of Triangular Wings at Supersonic Speeds", N.A.C.A. T.N. No. 1572 , 1948.

5. Moeckel, W. E. and Evvard, J. C., "Load Distributions Due to Steady Roll and Pitch for Thin Wings at Supersonic Speeds", N.A.C.A. T.N. No. 1689, 1948.

6. Malvestuto, F. S., Jr., and Margolis, K., "Theoretical Stability Derivatives of Thin Sweptback Wings Tapered to a Point with Sweptback and Sweptforward Trailing Edges for a Limited Range of Supersonic Speeds", N.A.C.A. T.N. No. 1761, 1949.

7. Miles, J. W., "On Damping in Pitch for Delta Wings", Journal of the Aeronautical Sciences, Vol. 16, No. 9, September 1949, pp. 574-576.

8. Moskowitz, B. and Moeckel, W. E., "First-Order Theory for Unsteady Motion of Thin Wings at Supersonic Speeds", N.A.C.A. T.N. No. 2034, 1950. 
No.

9. Stewart, H. J., and Li, Ting-Yi, "Periodic Motions of a Rectangular Wing Moving at Supersonic Speed", Journal of the Aeronautical Sciences, Vol. 17, No. 9, September 1950, pp. 529-539.

10. Miles, J. W., "Unsteady Flow Theory in Dynamic Stability", Journal of the Aeronautical Sciences, Vol. 17, No. 1, January 1950, pp. 62-63.

11. Prandtl, I. and Tietjens, O. G., "Fundamentals of Hydro- and Aeromechanics", lst edition, McGraw-Hill, New York, 1934, Chapter 10.

12. Campbell, G. A. and Foster, R. M., "Fourier Integrals for Practical Applications", Bell Telephone System Technical Publications, Monograph B-584, September 1931, p. 113.

13. Tsien, H., "Supersonic Flow Over an Inclined Body of Revolution", Journal of the Aeronautical Sciences, Vol. 5, No. 12, October 1938, pp. 480-484.

14. Laitone, E. V., "The Linearized Subsonic and Supersonic Flow About Inclined Slender Bodies of Revolution", Journal of the Aeronautical Sciences, Vol. 14, No. 11, November 1947, pp. 631642. 
WILLOW RUN RESEARCH CENTER - UNIVERSITY OF MICHIGAN

UMM-80

\section{DISTRIBUTION}

Distribution of this report is made

in accordance with ANAF-GM Mailing

List No. 14, dated January 15, 1951

to include Part A, Part B and Part C. 
